\title{
CAN SELECTIVE RETROPERITONEAL LYMPHADENECTOMY BE BETTER THAN UNILATERAL RETROPERITONEAL LYMPHADENECTOMY?
}

\author{
HOMERO O. ARRUDA, ADRIANO A.P. PAULA, RUBEN SUAREZ, JOSÉ CURY, \\ MIGUEL SROUGI \\ Division of Urology, Paulista School of Medicine, Federal University of São Paulo, São \\ Paulo, SP, Brazil
}

\begin{abstract}
Objective: To propose a new modality of retroperitoneal lymphadenectomy as a complementary treatment for patients with high risk, stage I nonseminomatous testicular tumor.

Materials and Methods: We studied 76 patients with stage I nonseminomatous testis tumor (T1-T4, NX, M0) treated by orchiectomy and retroperitoneal lymphadenectomy. Among them, 33 patients underwent unilateral retroperitoneal lymphadenectomy (URL) and 43 selective retroperitoneal lymphadenectomy (SRL). URL consisted in removing the lymph nodes located around the great vessel homolateral to the tumor (aorta or vena cava and iliac vessels), and anterior and posterior to the contralateral great vessel (aorta or vena cava). SRL was performed removing the lymph nodes located anterior and between the great vessels (aorta or vena cava) and laterally to the homolateral great vessel, extending the distal dissection until the level of inferior mesenteric artery. In these groups of patients, the incidence of disease recurrence, disease-free survival index, and frequency of post-operative aspermia were assessed. Mean post-operative follow-up time was 96 months.

Results: In the SRL group there was only 5\% of aspermia versus $79 \%$ in the URL group ( $\mathrm{p}<0.0001$ ). Tumor recurrence was observed in only 5 of the 76 patients and was not related to the surgical technique. The disease-free survival rate after the mean follow-up of 96 months was similar in both groups, being 94\% in the SRL group and 93\% in the URL group.

Conclusion: The selective retroperitoneal lymphadenectomy constitutes an effective technique with a lower morbidity than unilateral lymphadenectomy, representing an excellent option for the management of patients with high-risk, stage I nonseminomatous testis tumor.
\end{abstract}

Key words: testis; germ cell tumor, neoplasm metastasis, lymph node excision Int Braz J Urol. 2003; 29: 412-7

\section{INTRODUCTION}

Nonseminomatous testis tumors represent 40 to $50 \%$ of testicular neoplasms and differ from seminomas because they are more aggressive and poorly radiosensitive. Their treatment is basically surgery and/or chemotherapy, resulting in high cure indexes (1).

For patients with stage I disease, tumor confined to the scrotum, the classical treatment until 1980 was done by orchiectomy associated with bilateral retroperitoneal lymphadenectomy (2), a procedure 
with high morbidity, since it caused ejaculatory dysfunction in $75 \%$ of patients (3-5). Since the 80 's, several authors improved the surgical technique, always intending to decrease the morbidity caused by the surgery. Surgeons began to perform the unilateral retroperitoneal lymphadenectomy modified by Fossa et al. (5) and the more detailed, "nerve sparing" dissection proposed by Jewet et al. (6), in order to preserve the sympathetic nerve branches. These surgical modalities present the same oncologic results of radical surgery, although with a variable preservation of ejaculatory function between 62 and 88\% (7-11).

Considering these results, some groups proposed post-orchiectomy surveillance only for stage I cases, with the purpose of avoiding the surgical aggression and also, avoid the issue of aspermia $(12,13)$. However, due to the presence of occult metastases in the retroperitoneum, which cause false-negative results in the clinical staging, recurrence occurs in about $22-35 \%$ of patients maintained under surveillance $(8,14)$, who will require a subsequent salvage treatment. Even though patients with recurrence in retroperitoneum present full responses above $80 \%$ with chemotherapy, the validity of surveillance has been questioned due to some disadvantages. Those include, in addition to the need of subsequent chemotherapy in cases of recurrence, the anxiety generated in the patient and the high costs of tests required for follow-up. Thus, aiming to preserve the excellent results of a more extensive lymphadenectomy, to minimize its consequences and the drawbacks of exclusive surveillance, we began to perform the so-called selective retroperitoneal lymphadenectomy, whose results are presented here.

\section{MATERIALS AND METHODS}

In this study we retrospectively assess 76 patients with nonseminomatous germinal testis tumor, operated in the period from 1978 to 1994. Surgeries were performed both by transperitoneal laparotomy and by thoracophrenolaparotomy. Thirty-three of the $76(43 \%)$ patients with stage I disease, (T1-T4, NX, M0) underwent classic unilateral retroperitoneal lymphadenectomy (URL), between 1978 and 1986, and 43 of the $76(57 \%)$ were treated through the selective technique (SRL), in the period from 1986 to 1994, by the same surgeon. Patients' age ranged from 16 to 44 years and the median was 25 years. Among the 76 patients, 11 had embryonic carcinoma, 7 teratomas, 19 teratocarcinoma, 1 choriocarcinoma and 38 mixed tumors (Table-1).

URL was performed as described by Pizzocaro (4) and Fossa et al. (5). It consisted in removing the lymph nodes located around the great vessel homolateral to the tumor (aorta, vena cava and iliac vessels), anterior and posterior to the contralateral great vessel (aorta or vena cava), with the renal hilum being the upper limit. SRL without "nerve-sparing" dissection was defined as removal of the retroperitoneal lymph nodal chain that presents the renal hilum as its upper limit, the inferior mesenteric artery as the lower limit, and the lateral ones according to the side of the tumor. Right para-caval, pre-caval and superficial inter aorta-cava and pre-aortic lymph nodes were excised on the right side; para- and preaortic, superficial inter aorta-cava and pre-caval lymph nodes were removed on the left side, according to the Figure-1. Thus, the nerve fillets of the paravertebral sympathetic chain and hypogastric plexus, contralateral to the tumor side, were always preserved.

The occurrence of ejaculatory dysfunction was explored between the second and the third month post-operatively or after the conclusion of chemotherapy, in cases where this approach was used. After the surgical procedures, all patients were kept on surveillance for 2 years, consisting in dosage of $\alpha$ -

Table 1 - Distribution of the 76 patients, who underwent unilateral retroperitoneal lymphadenectomy (URL) and selective retroperitoneal lymphadenectomy (SRL), according to tumor histology.

\begin{tabular}{lcrr}
\hline Histology & URL & SRL & Total \\
\hline Embryonic Ca & 4 & 7 & 11 \\
Choriocarcinoma & 1 & 0 & 1 \\
Teratocarcinoma & 7 & 12 & 19 \\
Teratoma & 3 & 4 & 7 \\
Mixed & 18 & 20 & 38 \\
Total & 33 & 43 & 76 \\
\hline
\end{tabular}


E
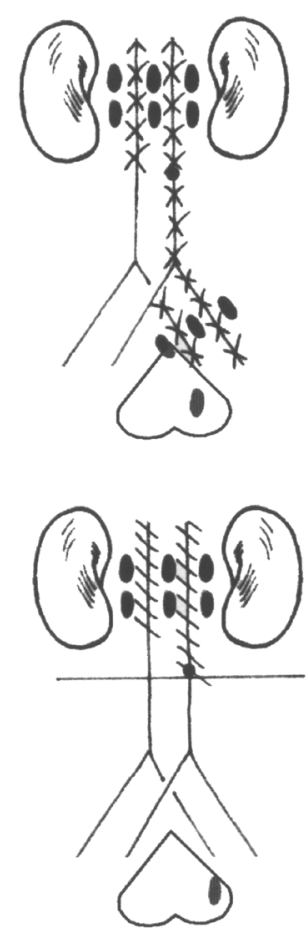

D

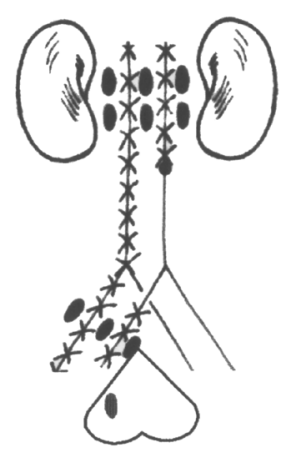

LRU

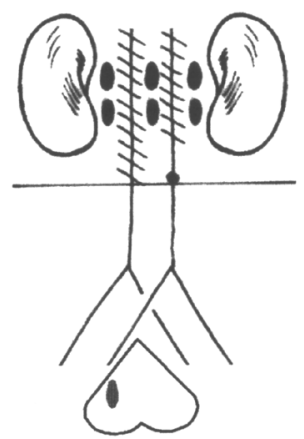

LRS

Figure 1 - Representation of unilateral retroperitoneal lymphadenectomy above (URL), and selective below (SRL), for tumors in the right $(R)$ and left $(L)$ side

fetoprotein and $\beta$-HCG markers every 3 months, and computerized axial tomography of chest and abdomen every 6 months. After this period, markers and image assessments were performed yearly. Adjuvant chemotherapy was performed whenever there was a compromised lymph node.

Table 2 - Distribution of the 76 patients who underwent unilateral retroperitoneal lymphadenectomy and selective retroperitoneal lymphadenectomy, according to pathologic staging, recurrence, and number of patients who were alive at the end of follow-up.

\begin{tabular}{lccc}
\hline Stage & $\mathbf{N}(\boldsymbol{\%})$ & Recurrence $(\boldsymbol{\%})$ & Alive $(\boldsymbol{\%})$ \\
\hline \multicolumn{1}{c}{ Unilateral Lymphadenectomy } & & \\
pT0 & $26(79)$ & 4 & 24 \\
pT1 & $7(21)$ & 0 & 7 \\
& $33(100)$ & $4(12)$ & $31(93)$ \\
& Selective & & 28 \\
pT0 & Unilateral Lymphadenectomy & 4 & 13 \\
pT1 & $30(70)$ & 1 & \\
\hline
\end{tabular}


Table 3 - Distribution of the 76 patients according to surgical technique of unilateral retroperitoneal lymphadenectomy $(U R L)$ or selective retroperitoneal lymphadenectomy (SRL) compared for the presence of aspermia $(p<0.0001)$.

\begin{tabular}{lcc}
\hline Techniques & Patients & Aspermia(\%) \\
\hline URL & 33 & $26(79)$ \\
SRL & 43 & $2(5)$ \\
Total & 76 & 28 \\
\hline
\end{tabular}

\section{RESULTS}

Seventy-six patients were retrospectively assessed as for the results of tumor recurrence, number of patients who were alive and disease-free, and the respective rates of ejaculatory dysfunction, following the 2 modalities of lymphadenectomy (Tables2 and 3). Both groups presented similar characteristics in relation to age and tumor histology. They differed only in the median of post-operative follow-up time: in the group who underwent unilateral lymphadenectomy (URL) it was between 66 and 168 months, and in the SRL group it was between 62 and 146 months. Of the 33 patients who underwent URL, $4(12 \%)$ presented systemic recurrence and were salvaged with chemotherapy. The follow-up in this group showed that 31 of the 33 (93\%) patients were alive and without evidence of disease, after the average time of 96 months. Among the 43 patients who underwent SRL, $13(30 \%)$ presented microscopic disease in retroperitoneum. Of these 13 patients, 12 underwent adjuvant chemotherapy. One patient did not accept it and was only observed as the others. The follow-up in this group (SRL) identified $5(11 \%)$ recurrences, with one occurring in abdomen, 3 systemic and one both abdominal and systemic, but none of them occurred below the mesenteric artery, and in 4, pathology did not detect disease in the removed lymph nodes. All patients who presented recurrence also received salvage chemotherapy and after the average time of 96 months, 41 patients out of 43 (95\%) were alive and with no evidences of neoplasia.
In relation to the aspermia rate, it was noted that in the group submitted to SRL it was only 5\%, versus $79 \%$ observed in the URL group (Table-3). No major surgical complication was observed among the groups.

\section{DISCUSSION}

In order to perform the lymphadenectomy as an adjuvant treatment for orchiectomy in nonseminomatous testis tumor, one must consider 2 fundamental biologic phenomena in relation to lymph nodal involvement: first, that lymphatic drainage is initially directed to lymph nodes of the renal hilum and from there, metastases dissemination occurs, cranially to the mediastinum and caudally towards the pelvis; second, that metastases initially involve lymph nodes located anterior to the great vessel homolateral to the testicular tumor, that is, from the right side to the region lateral to cava, anterior and between aorta and cava, and from the left side, anterior and lateral to aorta. Additionally, about $5 \%$ of tumors in the right testis and $10 \%$ of tumors in the left testis (13), develop metastasis in the contralateral renal hilum. For this reason, the lymphadenectomy adjuvant to orchiectomy in the treatment of testicular tumor should prioritize the removal of peri-hilar lymph nodes, including those in the contralateral renal hilum.

Due to this behavior of the neoplasia, early removal of potentially compromised lymph nodes can provide, in addition to the accurate staging of the disease, the possibility of cure for the patient even after the beginning of lymph nodal metastatic dissemination to the retroperitoneum. Following the description and mapping of metastatic regions described by Ray et al. in 1974 (15) and subsequently by Donohue et al. (16), surgeons started to increasingly perform less extensive procedures, aiming to decrease surgical morbidity. Such results were achieved in the follow-up of our 76 patients, of whom 93\% in the URL group and $95 \%$ in the SRL group were alive and with no evidence of disease, after the mean period of 96 months. We also confirmed the observations by other authors (17) that the lymphadenectomy, when performed through transperitoneal laparotomy, has the 
advantage of allowing bilateral dissection of lymph nodes, however, the approach to suprahilar ones is more difficult, when compared with thoracophrenolaparotomy. An easier approach to suprahilar lymph nodes is achieved by this route, with lower morbidity. Nevertheless, more important than the access route were the results relative to morbidity secondary to the strategy used for performing the surgery, which we will describe next.

At first, lymph nodal removal usually resulted in aspermia, due to lesion of the paravertebral ganglia, an extremely undesirable event, considering that these patients are young individuals and in reproductive age. And though the works by Fossa et al. (5) indicated that, when the modified unilateral lymphadenectomy was used, restricting bilateral dissection, a decrease in aspermia was achieved, this complication occurred in $60 \%$ of patients and was caused by lesion of the hypogastric plexus at the level of the aorta bifurcation . For these reason, we decided to use the lymphadenectomy in an even more selective way ("selective lymphadenectomy") than the already described one, discontinuing the dissection, in its lower limit, at the level of the inferior mesenteric artery, in order to preserve the sympathetic ganglia and to keep the patient's ejaculatory capacity. In fact, through the selective dissection of the lymphatic chain, we reduced our aspermia index from $79 \%$ to $5 \%$, evidencing the importance of maintaining the hypogastric plexus. An equally important result was that the reduction in aspermia was not accompanied by a higher index of tumor recurrence, proving its effectiveness as a curative treatment adjuvant to orchiectomy. In this study, the occult micrometastasis rate was nearly $30 \%$ in both groups, similarly to that observed in the literature $(7,10)$. One must emphasize that when lymph nodes were positive, adjuvant chemotherapy was performed, in order to induce full remission of disease, since from the oncologic point of view the lymph nodal removal could have been only partial.

A controversy we experienced as well and must be mentioned, is the fact that by performing surgery in all patients with stage I nonseminomatous tumor, maybe we were "over treating" $70 \%$ of the patients, which could be cured just with the orchiec- tomy. But, on the other hand, we were avoiding late recurrence, the patient's anxiety during the followup, and the high costs of subsidiary tests that are imposed to these patients, when an option for surveillance is made. In clinical practice an effective way of reducing the rate of unnecessary or ineffective surgeries, without failing to attend patients who would need surgery, is to include "non-reliable patients" among those with high risk for occult retroperitoneal disease (1). According to this criterion, patients who present factors of worse prognosis are selected for surgery, and those are: presence of vascular invasion, invasion of the cord and/or epididymis, extremely high tumor markers, presence of more than $50 \%$ of embryonic carcinoma or presence of yolk sac type tumor $(8,12,13,18)$ and also, patients whose clinical follow-up could be incomplete, or not performed in an assiduous and reliable way.

When comparing the aspermia rates obtained in our group of patients with those mentioned by other authors (7-9), we observed distinct results according to the different surgical techniques. Donohue et al. (8) reported keeping the ejaculation in only $9 \%$ of cases when the dissection was bilateral and extended to the suprahilar region, and in $18 \%$ when it was bilateral and infrahilar, and in $75 \%$ when the lymphadenectomy was exclusively unilateral, although with no preservation of the sympathetic plexus at the level of the bifurcation of the aorta. However, the "nerve-sparing" procedure with preservation of the critical segment of the hypogastric plexus was followed by preservation of ejaculation in almost every patient (9). In our cases, merely discontinuing the dissection above the emergence of the inferior mesenteric artery, with no need for a detailed identification of the nervous fibers to be spared, was enough to, in addition to decreasing the surgical time, provide the maintenance of sperm emission in $95 \%$ of our patients. In other words, we obtained an increase of $20 \%$ in the rate of patients who preserved ejaculation, with no impairment in disease-free survival rates, just by limiting the surgery's extension.

Thus we conclude that, according to our results, selective retroperitoneal lymphadenectomy in patients with stage I nonseminomatous testis tumor, represents a feasible option, that is simpler than the 
procedures performed up to now, requires a shorter surgical time and fundamentally, is followed by minimal ejaculatory dysfunction. In relation to oncologic results, the "selective lymphadenectomy" that we began to use, provided an accurate identification of patients who required adjuvant chemotherapy and presented results that are equivalent to those of unilateral lymphadenectomy, concerning the survival during the period of 96 months.

\section{REFERENCES}

1. Srougi M, Simon SD: Germ-cell Testicular Tumors. In: Srougi M, Simon SD (eds.), Urological Cancer. São Paulo, Marprint. 1989; pp. 377-433 [in Portuguese].

2. Donohue J: Retroperitoneal lymphadenectomy: the anterior approach including bilateral and suprahilar dissection. Urol Clin North Am. 1977; 4: 509-21.

3. Baniel J, Foster RS, Rowland RG. Bihrle R, Donohue JP: Complications of primary retroperitoneal lymph node dissection. J Urol. 1994; 152: 424-7.

4. Pizzocaro G: Retroperitoneal lymphadenectomy in clinical stage I nonseminomatous germinal testis cancer. Eur J Surg Oncol. 1986; 12: 25-8.

5. Fossa SD, Kleep O, Ous S, Lien HH, Stenwig AE, Abyholm T, et al.: Unilateral retroperitoneal lymph node dissection in patients with nonseminomatous testicular tumor in clinical stage I. Eur Urol. 1984; 10: 17-23.

6. Jewett MA, Kong YS, Goldberg SD, Sturgeon JF, Thomas GM, Alison RE, et al.: Retroperitoneal lymphadenectomy for testis tumor with nerve-sparing for ejaculation. J Urol. 1988; 139: 1220-4.

7. Foster RS, McNulty A, Rubin LR, Bennett R, Rowland RG, Sledge GW, et al.: The fertility of patients with clinical stage I testis cancer managed by nerve sparing retroperitoneal lymph node dissection. J Urol. 1994; 152: 1139-43.

8. Donohue JP, Thornhill JA, Foster RS, Rowland RG, Bihrle R: Retroperitoneal lymphadenectomy for clinical stage A testis cancer (1965-1989): modifications of technique and impact on ejaculation. J Urol. 1993; 149: 237-43.

9. Donohue JP, Foster RS, Rowland RG, Bihrle R, Jones J, Geier G: Nerve-sparing retroperitoneal lymphadenectomy with preservation of ejaculation. J Urol. 1990; 144: 287-92.

10. Walsh P, Kaufman J, Coulson W, Goodwin WE: Ret- roperitoneal Lymphadenectomy for testicular tumors. JAMA. 1971; 217: 309-12.

11. Johnson DE, Bracken RB, Blight EM: Prognosis for pathologic stage I non-seminomatous germ cell tumors of the testis managed by retroperitoneal lymphadenectomy. J Urol. 1976; 116: 63-5.

12. Kleep O, Dahl O, Flodgren P, Stierner U, Olssom AM, Oldbring J, et al.: Risk - adapted treatment of clinical stage 1 non - seminoma testis cancer. Eur J Cancer. 1997; 33: 1038-44.

13. Read G, Stenning SP, Cullen MH, Parkinson MC, Horwich A, Kaye SB, et al.: Medical Research Council prospective study of surveillance for stage I testicular teratoma. J Clin Oncol. 1992; 10: 1762-8.

14. Lange PH, Narayan P, Fraley EE: Fertility issues following therapy for testicular cancer. Semin Urol. 1984; 2: 264-74.

15. Ray B, Jewett MA, Donohue RE: Summary of distribution of retroperitoneal lymph node metastases in testicular germinal tumors. Semiun Urol Oncol. 1997; 15: 130-5.

16. Donohue JP, Zachary JM, Maynard BR: Distribution of nodal metastasis in nonseminomatous testis cancer. J Urol. 1982; 128: 315-20.

17. Wise PG, Scardino PT: Thoracoabdominal retroperitoneal lymphadenectomy for nonseminomatous testicular cancer. Urol Clin North Am. 1983; 110: 371-9.

18. Hoskin P, Dilly S, Easton D, Worwich A, Hendry W, Peckham MJ: Prognostic factors in stage I nonseminomatous germ-cell testicular tumors managed by orchiectomy and surveillance: Implications for adjuvant chemotherapy. J Clin Oncol. 1986; 4: 1031-6.

Received: April 25, 2003

Accepted after revision: August 4, 2003

\section{Correspondence address:}

Dr. Homero Oliveira de Arruda

Rua Napoleão de Barros, 715 / $2^{\circ}$ andar

São Paulo, SP, 04024-002, Brazil

Fax: + $55114521-9658$

E-mail: arrudas@dglnet.com.br 